Article

\title{
Exploring the Mechanical Anisotropy and Ideal Strengths of Tetragonal $\mathrm{B}_{4} \mathrm{CO}_{4}$
}

\author{
Baobing Zheng *, Meiguang Zhang and Canjun Wang \\ College of Physics and Optoelectronics Technology, Nonlinear Research Institute, \\ Baoji University of Arts and Sciences, Baoji 721016, China; zhmgbj@126.com (M.Z.); cjwangbj@126.com (C.W.) \\ * Correspondence: scu_zheng@163.com; Tel.: +86-917-3364-258
}

Academic Editor: Giorgio Biasiol

Received: 23 December 2016; Accepted: 26 January 2017; Published: 4 February 2017

\begin{abstract}
First-principles calculations were employed to study the mechanical properties for the recently proposed tetragonal $\mathrm{B}_{4} \mathrm{CO}_{4}\left(t-\mathrm{B}_{4} \mathrm{CO}_{4}\right)$. The calculated structural parameters and elastic constants of $t-\mathrm{B}_{4} \mathrm{CO}_{4}$ are in excellent agreement with the previous results, indicating the reliability of the present calculations. The directional dependences of the Young's modulus and shear modulus for $t-\mathrm{B}_{4} \mathrm{CO}_{4}$ are deduced in detail, and the corresponding results suggest that the $t-\mathrm{B}_{4} \mathrm{CO}_{4}$ possesses a high degree of anisotropy. Based on the strain-stress method, the ideal tensile and shear strengths along the principal crystal directions are calculated, and the obtained results indicate that the shear mode along (001)[100] slip system dominates the plastic deformation of $t-\mathrm{B}_{4} \mathrm{CO}_{4}$, which can be ascribed to the breaking of the ionic B-O bonds. The weakest ideal shear strength of $27.5 \mathrm{GPa}$ demonstrates that the $t-\mathrm{B}_{4} \mathrm{CO}_{4}$ compound is not a superhard material, but is indeed a hard material. Based on the atomic explanation that the ternary B-C-O compounds cannot acquire high ideal strength, we propose two possible routes to design superhard B-C-O compounds.
\end{abstract}

Keywords: anisotropic properties; ideal strengths; superhard; B-C-O compound

\section{Introduction}

Superhard materials, defined as materials with Vickers hardness higher than $40 \mathrm{GPa}$, are of great interest in many industrial areas, such as abrasives, polishing, cutting tools, and protective coatings. Generally, superhard materials require a high valence electron density and high bond covalency to form strong covalent bonds that would enhance their structural strength against large inelastic deformations, consequently leading to increased hardness. Therefore, the recent quest for intrinsic superhard materials mainly concentrates on two categories of compounds [1]. One category consists of the compounds formed by electron-rich transition-metal (TM) and small first row main group elements (e.g., boron, carbon, and nitrogen), such as $\mathrm{ReB}_{2}$ [2], $\mathrm{OsB}_{2}$ [3], $\mathrm{WB}_{4}$ [4,5], $\mathrm{FeB}_{4}$ [6], $\mathrm{Os}_{2} \mathrm{C}$ [7], $\mathrm{PtN}_{2}$ [8], $\mathrm{IrN}_{2}$ [9], etc. Although these compounds exhibit large bulk moduli close to that of diamond and are hence considered as ultra-incompressible materials, further experimental and theoretical studies suggest that most of the TM borides, carbide, and nitrides are unlikely to be superhard [7,10-13]. The other category consists of light element compounds (B-C-N-O) with strong covalent bonds, such as $\mathrm{B}-\mathrm{C}$ compounds $\left(\mathrm{BC}_{3}[14], \mathrm{BC}_{5}[15]\right), \mathrm{B}-\mathrm{C}-\mathrm{N}$ compounds $\left(\mathrm{BC}_{2} \mathrm{~N}\right.$ [16]), $\mathrm{B}-\mathrm{O}$ compounds $\left(\mathrm{B}_{6} \mathrm{O}\right.$ [17]), and B-C-O compounds.

In particular, the successful syntheses of the B-C-O system have spurred extensive research efforts in the study of those compounds due to their excellent mechanical properties [18-20]. However, the stoichiometric ratios of recently synthesized B-C-O systems under high pressures, which belong to the single crystal of the interstitial phases based on the rhombohedral $\alpha$-boron modification, are very complex. Garvie et al. [18] prepared a series of ternary phases in the B-C-O system with the mixtures of $\mathrm{B}, \mathrm{C}$, and $\mathrm{B}_{2} \mathrm{O}_{3}$ under pressure between 5 and $7.5 \mathrm{GPa}$ and a temperature of $1700{ }^{\circ} \mathrm{C}$. 
The boron suboxycarbide $\mathrm{B}(\mathrm{C}, \mathrm{O})_{0.155}$ single crystal has been successfully synthesized by chemical reaction between the $\mathrm{B}_{4} \mathrm{C}$ and $\mathrm{B}_{2} \mathrm{O}_{3}$ compounds under a pressure of $5.5 \mathrm{GPa}$ and a temperature of $1400 \mathrm{~K}$ [19]. Theoretically, the advent of density functional theory has provided us a powerful tool for theoretical studies when treating the energetics/structure/bonding of carbon/boron-based systems $[7,12,13,21-23]$. Moreover, the vigorous development of crystal structure prediction methods makes it possible to efficiently design functional materials that only require the chemical compositions. Based on the particle swarm optimization technique, the potential superhard compound ${ } \mathrm{BC}_{2} \mathrm{O}$ in the B-C-O system is proposed with theoretical Vickers hardness of 50 GPa [24]. Recently, Liu et al. predicted a lonsdaleite-like orthorhombic structure $\mathrm{B}_{2} \mathrm{CO}$ with strong $s p^{3}$ covalent $\mathrm{B}-\mathrm{C}$ and $\mathrm{B}-\mathrm{O}$ bonds [25]. Very recently, we studied the higher carbon content in ternary $\mathrm{B}_{2} \mathrm{C}_{\mathrm{x}} \mathrm{O}$ compounds which are isoelectronic with diamond, in order to search for novel superhard materials [26]. However, the three ultra-incompressible and thermodynamically stable $\mathrm{B}_{2} \mathrm{C}_{\mathrm{x}} \mathrm{O}$ compounds possess substantially lower ideal shear strengths than those of diamond and $c-\mathrm{BN}$, suggesting that they may not be intrinsically superhard in spite of the calculated hardness being higher than $40 \mathrm{GPa}$. Using the evolutionary algorithm, Wang et al. [27] explored a new tetragonal thermodynamically stable phase $\mathrm{B}_{4} \mathrm{CO}_{4}$ (space group $I \overline{4})$ with a claimed hardness of $\sim 40 \mathrm{GPa}$, indicating that the tetragonal $\mathrm{B}_{4} \mathrm{CO}_{4}\left(t-\mathrm{B}_{4} \mathrm{CO}_{4}\right)$ is potentially superhard. Intuitively speaking, despite its tetrahedrally coordinated $\mathrm{B}$ and $\mathrm{C}$ atoms, the presence of weak $\mathrm{B}-\mathrm{O}$ bonds and the large hollow space in the center of the $t-\mathrm{B}_{4} \mathrm{CO}_{4}$ structure suggest that whether or not $t-\mathrm{B}_{4} \mathrm{CO}_{4}$ is a superhard material needs to be further clarified.

In the present work, using the first-principles calculations, we systematically investigated the mechanical properties of the $t-\mathrm{B}_{4} \mathrm{CO}_{4}$ in comparison with other B-C-O structures. The formulas of the Young's and shear moduli along the arbitrary directions for Laue class $4 / m$ of the tetragonal crystal system were deduced, in order to study their elastic anisotropy. The ideal strengths were estimated to clarify whether the $t-\mathrm{B}_{4} \mathrm{CO}_{4}$ is superhard and to provide an atomic explanation of its plastic deformation.

\section{Computational Methods}

Using density functional theory with the Perdew-Burke-Ernzerhof (PBE) exchange correlation, we carried out the geometry optimization and the total energy calculations as implemented in the Vienna ab initio Simulation Package (VASP) [28,29]. The projector augmented-wave (PAW) method combined with the frozen core approximation was employed to describe the electron and core interactions [30], where the $2 s^{2} 2 p^{1}, 2 s^{2} 2 p^{2}$, and $2 s^{2} 2 p^{4}$ are considered as valence electrons for $\mathrm{B}$, $\mathrm{C}$, and $\mathrm{O}$, respectively. The total-energy and elastic constants were calculated with a $550 \mathrm{eV}$ plane-wave cutoff energy and a grid of $0.03 \AA^{-1}$ Monkhorst-Pack $k$ point meshes [31], which are accurate enough to ensure that the enthalpy results were well converged to below $1 \mathrm{meV} / \mathrm{f}$.u. The successfully utilized strain-stress method was introduced to evaluate the elastic constants. The bulk modulus, shear modulus, and Young's modulus were calculated via the Voigt-Reuss-Hill approximation [32]. We applied continuous deformation upon the $t-\mathrm{B}_{4} \mathrm{CO}_{4}$ cell by increasing the displacement in the direction of the corresponding strain, and then estimated the ideal tensile and shear strengths from the yield stress $[33,34]$. The convergence tests indicate that the calculated elastic constants and ideal strengths are almost independent of the energy cutoff, $k$-point mesh, and smearing parameter (see Supplemental Material).

\section{Results and Discussion}

The optimized structures of $t-\mathrm{B}_{4} \mathrm{CO}_{4}$ along the [001] and [010] view directions are shown in Figure 1. Each $C$ atom is coordinated with four $B$ atoms, and each $B$ atom is coordinated with one $C$ atom and three $\mathrm{O}$ atoms. All the lengths of the $\mathrm{B}-\mathrm{C}$ bonds are $1.571 \AA$, and the bond lengths of the three kinds of $\mathrm{B}-\mathrm{O}$ bonds are $1.505 \AA, 1.543 \AA, 1.569 \AA$, respectively, which is consistent with the previous results of the average B-C and B-O bond lengths of $1.570 \AA$ and $1.539 \AA$ at ambient pressure [27]. The slight differences between our estimated values and previous results suggest that the present calculations are accurate and reliable. The calculated elastic constants $C_{i j}$, bulk modulus $B$, shear modulus $G$, and Young's modulus $G$ at ambient pressure are summarized in Table 1 together with other B-C-O systems for comparison. 
Table 1. Calculated Elastic Constants $C_{i j}$, Bulk Modulus $B$, Shear Modulus $G$, Young's Modulus $E$, and ideal strength (minimum tensile strength $\sigma_{m i n}$ and shear strength $\tau_{\text {min }}$ ) of $t-\mathrm{B}_{4} \mathrm{CO}_{4}$ together with other ternary B-C-O compounds (in units of GPa).

\begin{tabular}{|c|c|c|c|c|c|c|c|c|c|c|c|c|c|}
\hline Compounds & Source & $C_{11}$ & $C_{33}$ & $C_{44}$ & $C_{66}$ & $C_{12}$ & $C_{13}$ & $C_{16}$ & $B$ & $G$ & $E$ & $\sigma_{\min }$ & $\tau_{\min }$ \\
\hline \multirow[t]{2}{*}{$t-\mathrm{B}_{4} \mathrm{CO}_{4}$} & This work & 481 & 452 & 269 & 260 & 150 & 129 & \multirow[t]{11}{*}{-52} & 248 & 218 & 505 & \multirow[t]{3}{*}{$\sigma_{[011]}=29.0$} & \multirow[t]{3}{*}{$\tau_{(001)[100]}=27.5$} \\
\hline & Theory ${ }^{1}$ & 480 & 449 & 268 & 259 & 152 & 131 & & 248 & 220 & 509 & & \\
\hline $\mathrm{tI} 16-\mathrm{B}_{2} \mathrm{CO}$ & Theory $^{2}$ & 600 & 646 & 304 & 283 & 182 & 144 & & 310 & 265 & & & \\
\hline $\mathrm{tP} 4-\mathrm{B}_{2} \mathrm{CO}$ & Theory $^{3}$ & 736 & 591 & 240 & 254 & 53 & 157 & & 311 & 254 & & \multirow[t]{2}{*}{$\sigma_{[111]}=6.1$} & \multirow[t]{2}{*}{$\tau_{(111)[1 \overline{1} 0]}=2.6$} \\
\hline $\mathrm{oP} 8-\mathrm{B}_{2} \mathrm{CO}$ & Theory 4 & 732 & 675 & 238 & 260 & 112 & 69 & & 298 & 270 & & & \\
\hline $\mathrm{B}_{2} \mathrm{C}_{2} \mathrm{O}$ & Theory $^{5}$ & 763 & 590 & 229 & 174 & 15 & 135 & & 299 & 264 & 611 & $\sigma_{[111]}=21.3$ & $\tau_{(111)[1 \overline{1} 0]}=11.1$ \\
\hline $\mathrm{B}_{2} \mathrm{C}_{3} \mathrm{O}$ & Theory ${ }^{5}$ & 808 & 664 & 283 & 299 & 32 & 183 & & 322 & 302 & 690 & $\sigma_{[111]}=22.4$ & $\tau_{(111)[1 \overline{1} 0]}=11.4$ \\
\hline $\mathrm{B}_{2} \mathrm{C}_{5} \mathrm{O}$ & Theory $^{5}$ & 889 & 740 & 346 & 335 & 30 & 135 & & 345 & 351 & 787 & $\sigma_{[111]}=25.1$ & $\tau_{(111)[1 \overline{1} 0]}=16.3$ \\
\hline $\mathrm{B}_{2} \mathrm{O}$ & Theory $^{2}$ & 327 & 497 & 232 & 207 & 230 & 144 & & 242 & 124 & & & \\
\hline$c-\mathrm{BN}$ & Theory ${ }^{6}$ & 786 & & 445 & & 172 & & & 376 & 390 & & $\sigma_{[111]}=55.3$ & $\tau_{(111)[11 \overline{2}]}=58.3$ \\
\hline Diamond & Theory ${ }^{7}$ & 1052 & & 555 & & 122 & & & 432 & 517 & 1109 & $\sigma_{[111]}=82.3$ & $\tau_{(111)[11 \overline{2}]}=86.8$ \\
\hline
\end{tabular}

${ }^{1}$ Ref. [27]; ${ }^{2}$ Ref. [24]; ${ }^{3}$ Ref. [24,26]; ${ }^{4}$ Ref. [25]; ${ }^{5}$ Ref. [26]; ${ }^{6}$ Ref. [35]; ${ }^{7}$ Ref. [36,37]. 


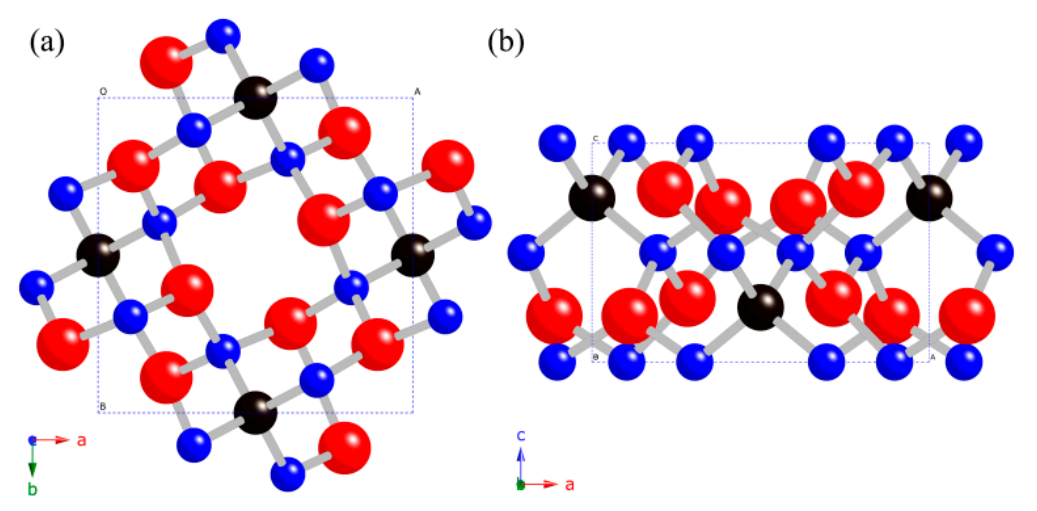

Figure 1. Crystal structures of $t-\mathrm{B}_{4} \mathrm{CO}_{4}$ as viewed along the [001] direction (a) and the [010] direction (b), the red, blue, and black spheres represent $\mathrm{O}, \mathrm{B}$, and $\mathrm{C}$ atoms, respectively.

For a tetragonal $I \overline{4}$ crystal, the seven $C_{i j}$ should satisfy the following necessary and sufficient stability conditions according to the Born stability criterion [38]:

$$
\begin{aligned}
& C_{11}>\left|C_{12}\right|, 2 C_{13}^{2}<C_{33}\left(C_{11}+C_{12}\right), \\
& C_{44}>0,2 C_{16}^{2}<C_{66}\left(C_{11}-C_{12}\right) .
\end{aligned}
$$

Clearly, the calculated elastic constants of $t-\mathrm{B}_{4} \mathrm{CO}_{4}$ meet all the mechanical stability criteria, indicating the mechanical stability of $t-\mathrm{B}_{4} \mathrm{CO}_{4}$. Compared with the other $\mathrm{B}-\mathrm{C}-\mathrm{O}$ systems from Table 1 , we find that the $C_{11}$ and $C_{33}$ of $t-\mathrm{B}_{4} \mathrm{CO}_{4}$ are significantly less than those of $\mathrm{B}_{2} \mathrm{CO}, \mathrm{B}_{2} \mathrm{C}_{2} \mathrm{O}, \mathrm{B}_{2} \mathrm{C}_{3} \mathrm{O}$, and $\mathrm{B}_{2} \mathrm{C}_{5} \mathrm{O}$, as well as the superhard $c-\mathrm{BN}$ and diamond, suggesting its lower incompressibility along the $a$ - and $c$-direction. The bulk modulus (248 GPa) and shear modulus (218 GPa) of $t-\mathrm{B}_{4} \mathrm{CO}_{4}$ are not only much smaller than those of typical superhard $c$-BN and diamond, but are also much smaller than those of the ternary B-C-O system. Although the plastic hardness is essentially inequivalent to the elastic modulus, the value of the elastic modulus indirectly reflects the hardness of the material. Thus, the noticeable difference of elastic moduli between $t-\mathrm{B}_{4} \mathrm{CO}_{4}$ and superhard $c$ - $\mathrm{BN}$ or diamond suggest that the superhard feature of $t-\mathrm{B}_{4} \mathrm{CO}_{4}$ should be further debated.

Anisotropy is a measure of a material's directional dependence of its physical or mechanical properties. Although all the known crystals are elastically anisotropic due to the atomic arrangement of the crystal structure of the material, the elastic anisotropy of crystal can actually play a dominant role in plastic deformation, crack behavior, and elastic instability when the anisotropy of a single crystal is large and crucial. Therefore, we systematically investigated the elastic anisotropy of the $t-\mathrm{B}_{4} \mathrm{CO}_{4}$ compound for its potential engineering applications. For a tetragonal $I \overline{4}$ structure, the Young's modulus for tensile stress along an arbitrary $[\mathrm{hkl}]$ direction can be expressed as the following equation:

$$
\begin{aligned}
& E^{-1}=s_{11}\left(\alpha^{4}+\beta^{4}\right)+s_{33} \gamma^{4}+2 s_{12} \alpha^{2} \beta^{2}+2 s_{13}\left(\beta^{2} \gamma^{2}+\alpha^{2} \gamma^{2}\right) \\
& +s_{44}\left(\beta^{2} \gamma^{2}+\alpha^{2} \gamma^{2}\right)+s_{66} \alpha^{2} \beta^{2}+2 s_{16} \alpha \beta\left(\alpha^{2}-\beta^{2}\right)
\end{aligned}
$$

where $\alpha, \beta$, and $\gamma$ are the direction cosines of the tensile stress direction deduced from the transformed coordinate system with respect to the original coordinate system, and $s_{11}, s_{12}, s_{13}, s_{33}, s_{44}, s_{66}$, and $s_{16}$ are the independent elastic compliance constants given by Kelly et al. [39], which are determined from the calculated elastic constants $C_{i j}$. For the Laue class $4 / m$ of the tetragonal crystal system, the extra elastic constant $C_{16}$ brings the total number of independent elastic compliances $s_{i j}$ to seven:

$$
\begin{aligned}
& s_{11}=s_{22}=\frac{1}{2}\left(\frac{C_{33}}{C^{\prime}}+\frac{C_{66}}{C^{\prime \prime}}\right), s_{12}=\frac{1}{2}\left(\frac{C_{33}}{C^{\prime}}-\frac{C_{66}}{C^{\prime \prime}}\right), s_{13}=-\frac{C_{13}}{C^{\prime}}, s_{33}=\frac{C_{11}+C_{12}}{C^{\prime}} \\
& s_{44}=\frac{1}{C_{44}}, s_{66}=\frac{C_{11}-C_{12}}{C^{\prime \prime}}, s_{16}=-\frac{C_{16}}{C^{\prime \prime}}
\end{aligned}
$$


where

$$
C^{\prime}=C_{33}\left(C_{11}+C_{12}\right)-2 C_{13}^{2}, C^{\prime \prime}=C_{66}\left(C_{11}-C_{12}\right)-2 C_{16}^{2}
$$

The analytical formulas of the Young's moduli for the tensile axis within specific planes, such as the (001), (100), and (11) tensile planes, are deduced from Equation (2) and are then listed in Table 2, where $\theta$ is the angle between the principal crystal direction of the tensile plane and the tensile stress direction.

Table 2. Formulas of Young's moduli for the tensile axis within specific planes.

\begin{tabular}{ccc}
\hline Tensile Plane & $\boldsymbol{E}^{-\mathbf{1}}$ & Orientation Angle $\boldsymbol{\theta}$ \\
\hline$(001)$ & $s_{11}-\frac{1}{4}\left(2 s_{11}-2 s_{12}-s_{66}\right) \sin ^{2} 2 \theta+s_{16} \sin 2 \theta\left(\sin ^{2} \theta-\cos ^{2} \theta\right)$ & between [hk0] and [100] \\
$(100)$ & $s_{11} \sin ^{4} \theta+s_{33} \cos ^{4} \theta+\frac{1}{4}\left(2 s_{13}+s_{44}\right) \sin ^{2} 2 \theta$ & between [0kl] and [001] \\
$(1 \overline{1} 0)$ & $\frac{1}{4}\left(2 s_{11}+2 s_{12}+s_{66}\right) \sin ^{4} \theta+s_{33} \cos ^{4} \theta+\frac{1}{4}\left(2 s_{13}+s_{44}\right) \sin ^{2} 2 \theta$ & between [hkl] and [001] \\
\hline
\end{tabular}

The three-dimensional (3D) surface representation, the three-plane projection drawings, and the orientation dependences in polar coordinates of the Young's modulus $E$ are plotted in Figure $2 \mathrm{a}-\mathrm{c}$, based on the analytical formulas in Table 2, respectively. The shape of the 3D surface representation far from sphere suggests that the Young's modulus of $t-\mathrm{B}_{4} \mathrm{CO}_{4}$ possesses a high degree of anisotropy. The projection drawing of the $a c$ and $b c$ planes for the $3 \mathrm{D}$ surface representation coincide with each other due to the symmetry of $t-\mathrm{B}_{4} \mathrm{CO}_{4}$. Interestingly, unlike the Laue class $4 / \mathrm{mmm}$ of the tetragonal crystal, the extra elastic constant $C_{16}$ of $t-\mathrm{B}_{4} \mathrm{CO}_{4}$ tilts the projection graph of the $a b$ plane. From Figure $2 \mathrm{c}$, we can conclude that the sequence of Young's moduli along the principal crystal directions is as follows: $E_{[010]}<E_{[001]}<E_{[011]}<E_{[110]}<E_{[111]}$. Note that the maximum Young's modulus in the principal crystal directions appears in the [111] direction, which shows a satisfactory agreement with the fact that the covalent $\mathrm{B}-\mathrm{C}$ bonds with high bond strength for $t-\mathrm{B}_{4} \mathrm{CO}_{4}$ mainly distribute in the [111] direction.

(a)

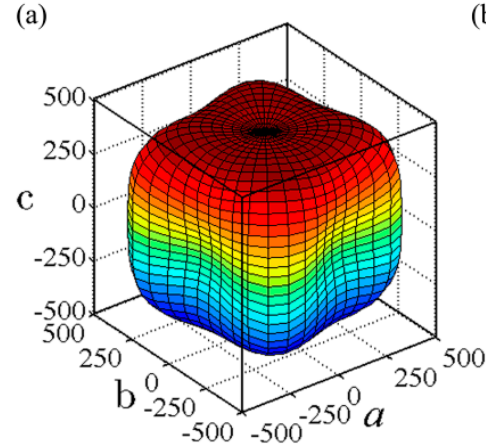

(c) (001) plane from $[100]$ to $[010]$
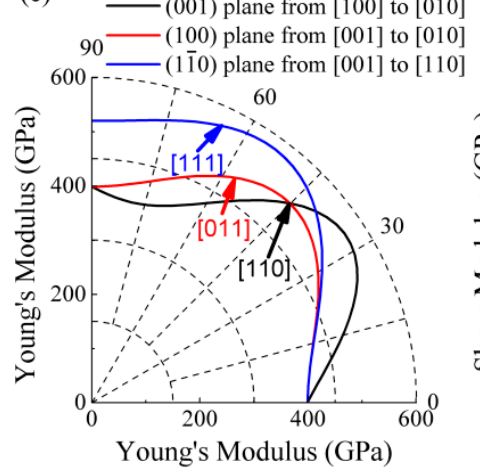

(b)
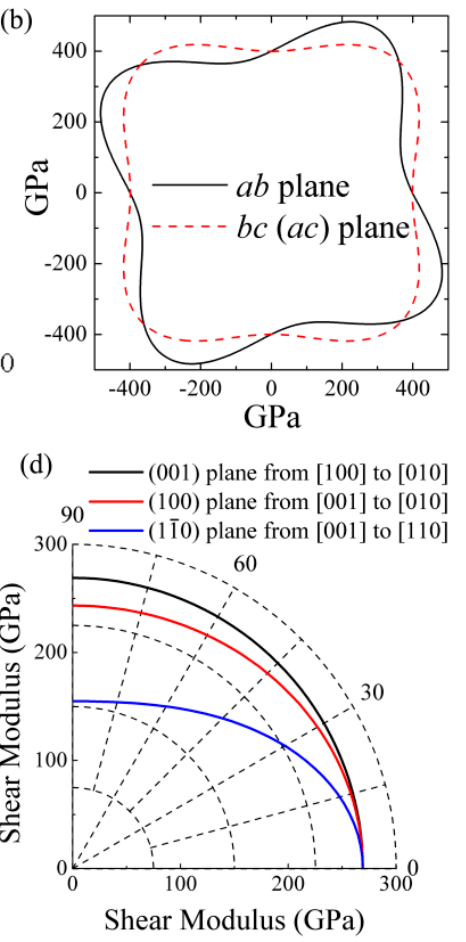

Figure 2. Orientation dependence of Young's Modulus $E(\mathbf{a}, \mathbf{c})$ and the corresponding projection in the $a b, a c$, and $b c$ planes (b) for the $t-\mathrm{B}_{4} \mathrm{CO}_{4}$, orientation dependence of the shear modulus of $t-\mathrm{B}_{4} \mathrm{CO}_{4}(\mathbf{d})$. 
The shear modulus $G$ on the $(h k l)$ shear plane with shear stress applied along the [uvw] direction is given by

$$
\begin{aligned}
& G^{-1}=4 s_{11}\left(\alpha_{1}^{2} \alpha_{2}^{2}+\beta_{1}^{2} \beta_{2}^{2}\right)+4 s_{33} \gamma_{1}^{2} \gamma_{2}^{2}+8 s_{12} \alpha_{1} \alpha_{2} \beta_{1} \beta_{2}+s_{66}\left(\alpha_{1} \beta_{2}+\alpha_{2} \beta_{1}\right)^{2} \\
& +8 s_{13}\left(\beta_{1} \beta_{2} \gamma_{1} \gamma_{2}+\alpha_{1} \alpha_{2} \gamma_{1} \gamma_{2}\right)+s_{44}\left[\left(\beta_{1} \gamma_{2}+\beta_{2} \gamma_{1}\right)^{2}+\left(\alpha_{1} \gamma_{2}+\alpha_{2} \gamma_{1}\right)^{2}\right] \\
& +4 s_{16}\left(\alpha_{1} \beta_{2}+\beta_{1} \alpha_{2}\right)\left(\alpha_{1} \alpha_{2}-\beta_{1} \beta_{2}\right)
\end{aligned}
$$

where $\left(\alpha_{1}, \beta_{1}, \gamma_{1}\right)$ and $\left(\alpha_{2}, \beta_{2}, \gamma_{2}\right)$ are the direction cosines of the [uvw] and $[H K L]$ directions in the primitive coordinate system, respectively, and the $[H K L]$ directions denote the vector normal to the $(h k l)$ shear plane. For a given shear plane, Equation (5) can be further simplified according to the orientation angle $\theta$ between the shear stress direction and the specified crystal direction. The deduced formulas of shear moduli along the (001), (100), and (11̄0) shear planes are summarized in Table 3. The orientation dependences of the shear modulus of $t-\mathrm{B}_{4} \mathrm{CO}_{4}$ are hence plotted in Figure $2 \mathrm{~d}$ for the shear (001), (100), and (11) planes. It is clear that the shear modulus within the (001) basal plane is independent of the orientation angle $\theta$, which results from the fact that the analytical formula of the shear modulus within the (001) basal plane is $G_{(001)}=\frac{1}{s_{44}}=C_{44}=269 \mathrm{GPa}$. On the other hand, the shear moduli within the (100) and (110) basal planes gradually decrease with the increase of the orientation angle $\theta$.

Table 3. Formulas of shear moduli for the shear stress direction within specific planes.

\begin{tabular}{ccc}
\hline Shear Plane & $G^{-1}$ & Orientation Angle $\boldsymbol{\theta}$ \\
\hline$(001)$ & $s_{44}$ & between [uvw] and [100] \\
$(100)$ & $s_{66}+\left(s_{44}-s_{66}\right) \cos ^{2} \theta$ & between [uvw] and [001] \\
$(1 \overline{1} 0)$ & $2\left(s_{11}-s_{12}\right) \sin ^{2} \theta+s_{44} \cos ^{2} \theta$ & between [uvw] and [001] \\
\hline
\end{tabular}

The hardness of $t-\mathrm{B}_{4} \mathrm{CO}_{4}$ estimated by the Lyakhov-Oganov model and the Chen-Niu model are $39 \mathrm{GPa}$ and $38 \mathrm{GPa}$ [24], respectively, which are in excellent agreement with each other, and are both close to $40 \mathrm{GPa}$. The Lyakhov-Oganov model for the Knoop hardness can be described by the following formula [40]:

$$
H_{k}(\mathrm{GPa})=\frac{423.8}{V} n\left[\prod_{k=1}^{n} N_{k} X_{k} e^{-2.7 f_{k}}\right]^{1 / n}-3.4,
$$

where $N_{k}$ is the number of bonds of type $k$ in the unit cell, and $X_{k}$ and $f_{k}$ are the electron-holding energy of the bond $k$ relevant to the electronegativities of atoms and its ionicity indicator, respectively. The Chen-Niu model can be summarized as follows [41]:

$$
H_{V}(\mathrm{GPa})=2\left(k^{2} G\right)^{0.585}-3, k=G / B
$$

Clearly, the two models based on either the elastic moduli or the electronegativity and covalent radii are actually not appropriate to describe the plastic hardness of a material. Physically, the indentation hardness in an experiment is measured under the condition of fully developed plasticity [42], and the plastic deformation of materials usually occurs far from equilibrium. However, all the introduced parameters in the two models are obtained under the equilibrium structure, which suggests that we should further check the superhard feature of $t-\mathrm{B}_{4} \mathrm{CO}_{4}$ employing other appropriate criteria. The ideal strengths, in particular the ideal shear strength, describe the resistance of the system at the atomic level where plastic deformation occurs, and thus are more suitable for assessing the possibility that a material may be superhard [42].

Here, we deform the $t-\mathrm{B}_{4} \mathrm{CO}_{4}$ cell gradually in the direction of the applied strain and then obtain the ideal strengths when the cell becomes mechanically unstable. Figure $3 a, b$ illustrates 
the calculated strain-stress relations under tensile and shear stress along the specified directions, respectively. The estimated tensile strengths in the [001], [011], [100], [110], and [111] directions are 32.2, 29.5, 42.6, 47.1, and 35.0 GPa, respectively. As listed in Table 1, the weakest tensile strengths of other B-C-O compounds except $t \mathrm{P} 4-\mathrm{B}_{2} \mathrm{CO}$ are quantitatively comparable to that of $t-\mathrm{B}_{4} \mathrm{CO}_{4}$, which occurs in the [011] tensile direction. Compared to diamond (82.3 GPa) and c-BN (55.3 GPa), the minimum tensile strength of $t-\mathrm{B}_{4} \mathrm{CO}_{4}(29.5 \mathrm{GPa})$ is not only lower than that of superhard diamond but is also lower than superhard $c$-BN. Moreover, the largest tensile strength of $t-\mathrm{B}_{4} \mathrm{CO}_{4}$ along the principal crystal direction is only $47.1 \mathrm{GPa}$, indicating the weak ability of the tensile resistance and the tendency to fracture under relatively small tensile stress for $t-\mathrm{B}_{4} \mathrm{CO}_{4}$.
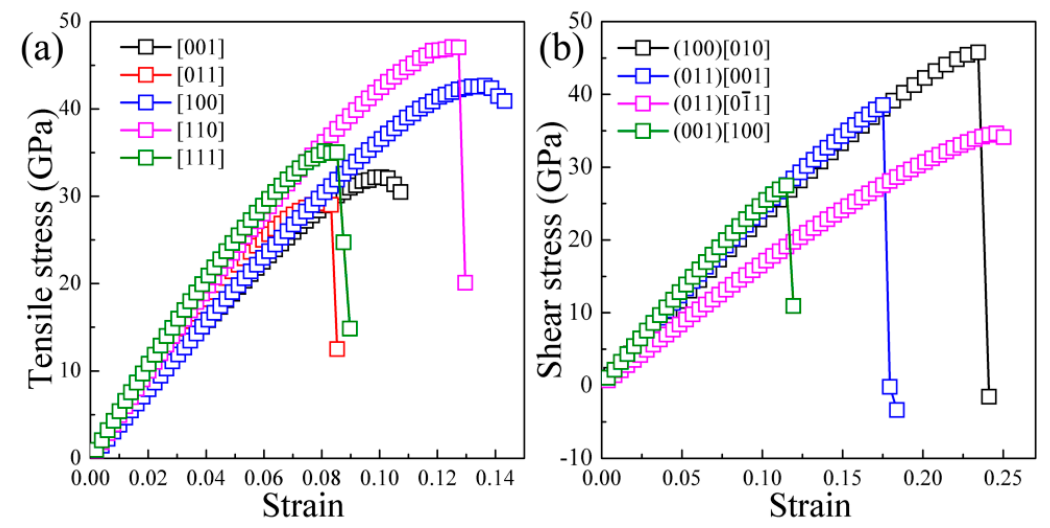

Figure 3. Calculated stress-strain relations for $t-\mathrm{B}_{4} \mathrm{CO}_{4}$ in various tensile (a) and shear (b) directions.

Generally, plastic deformation occurs in shear, so the ideal shear strength is more suitable for measuring whether a material may be superhard rather than theoretical hardness calculated by semi-empirical or first-principles methods. The lowest ideal shear strength of 27.5 GPa is found along the (001)[100] slip system for $t-\mathrm{B}_{4} \mathrm{CO}_{4}$. This value is markedly lower than the minimum shear strengths of $c$-BN (58.3 GPa) and diamond (86.8 GPa), but higher than those of other B-C-O compounds (see Table 1). The value of $27.5 \mathrm{GPa}$ is much less than the superhard criterion of $40 \mathrm{GPa}$, and suggests that the $t-\mathrm{B}_{4} \mathrm{CO}_{4}$ compound is therefore intrinsically hard, but not superhard as we expected. Note that the minimum shear strength is smaller than the minimum tensile strength. Thus, the shear mode in the (001)[100] slip system dominates the plastic deformation of $t-\mathrm{B}_{4} \mathrm{CO}_{4}$ rather than the tensile mode.

Figure $3 b$ shows a sharp decrease at a critical strain of $\gamma=0.1151$ along the (001)[100] slip system, implying a lattice instability for $t-\mathrm{B}_{4} \mathrm{CO}_{4}$. We next explored the atomic explanation for the plastic structural deformation of $t-\mathrm{B}_{4} \mathrm{CO}_{4}$ along the (001)[100] slip system. The Bader charge analysis revealed that the numbers of Bader charges for the $\mathrm{B}$ and $\mathrm{O}$ atoms are $2.80 e$ and $9.57 e$ in $t-\mathrm{B}_{4} \mathrm{CO}_{4}$, respectively, suggesting the ionic feature of $\mathrm{B}-\mathrm{O}$ bonds. Furthermore, as plotted in Figure 4, the isosurface of the electron localization function (ELF) for $t-\mathrm{B}_{4} \mathrm{CO}_{4}$ shows the valence electrons of $\mathrm{B}-\mathrm{O}$ bonds are highly localized around the $\mathrm{O}$ atoms, which further confirms the ionic feature of $\mathrm{B}-\mathrm{O}$ bonds. Figure 4 also illustrates the structural transformation of the $t-\mathrm{B}_{4} \mathrm{CO}_{4}$ before and after the lattice instability, i.e., at a shear strain of $\gamma=0.1109$ and $\gamma=0.1193$. Clearly, although the B-C bonds in $t-\mathrm{B}_{4} \mathrm{CO}_{4}$ do not present significant changes, the lengths of part of the B-O bonds (denoted as double-arrow lines) increase visibly from $1.724 \AA$ to $2.364 \AA$ before and after the lattice instability. It is well known that the strength of an ionic bond is much weaker than that of a covalent bond, which indicates the lattice instability of $t-\mathrm{B}_{4} \mathrm{CO}_{4}$ may result from the breaking of $\mathrm{B}-\mathrm{O}$ bonds rather than $\mathrm{B}-\mathrm{C}$ bonds. A bond critical point $(\mathrm{BCP})$ analysis revealed that the charge density at the $\mathrm{B}-\mathrm{O}$ bond critical point decreases from 0.624 electrons $/ \AA^{3}$ to 0.183 electrons $/ \AA^{3}$ when the shear strain increases from $\gamma=0.1109$ to $\gamma=0.1193$. Meanwhile, the Laplacian value decreases significantly from 2.973 to 1.231 . All these results support the fact that the lattice instability of $t-\mathrm{B}_{4} \mathrm{CO}_{4}$ under shear load originates from the breaking of B-O bonds. 


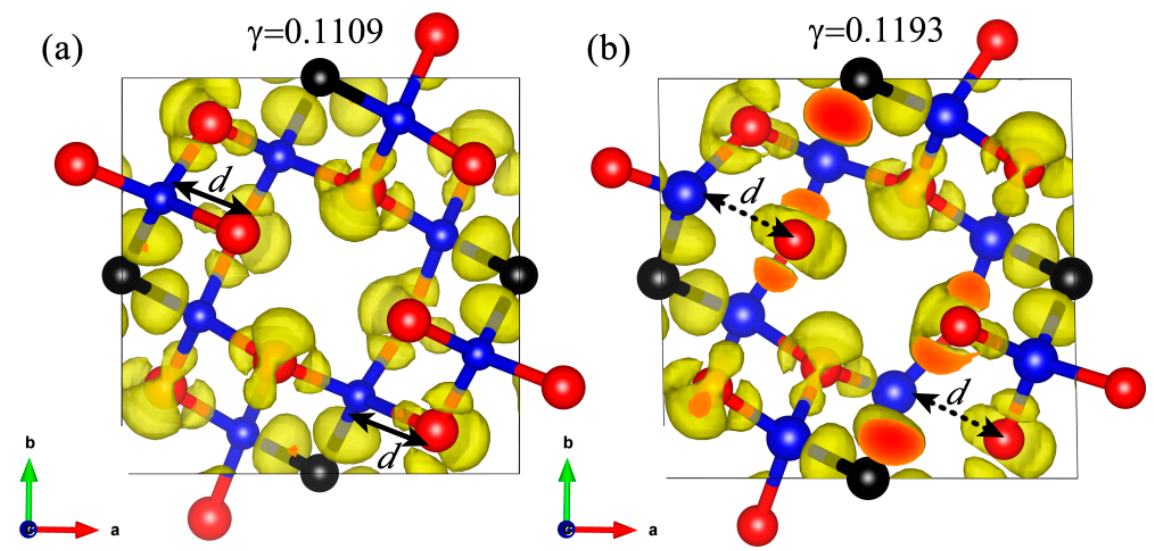

Figure 4. Structural and ELF transformation before (a) and after (b) the lattice instability for $t-\mathrm{B}_{4} \mathrm{CO}_{4}$.

It is well known that the lattice instability of the material is directly relevant to the imaginary modes of phonon dispersion of this material. To really understand the origin of the lattice instability and the key mechanisms that dominate the shear deformation, we calculated the phonon dispersion of $t-\mathrm{B}_{4} \mathrm{CO}_{4}$ at the shear strain of $\gamma=0.1193$ in the (001)[100] slip system. The obtained dispersion curves by the finite displacement method before and after shear deformation are plotted in Figure 5. Clearly, imaginary frequencies are found near the $\Gamma$ point, and the most unstable modes occur at the $(0,0,0.05)$ point along the $\Gamma$ to $\mathrm{Z}$ direction, which suggests that the lattice of $t-\mathrm{B}_{4} \mathrm{CO}_{4}$ has become unstable at the large shear deformation. Further analysis of the eigenvectors for the largest imaginary phonon modes indicates that the atoms of $t-\mathrm{B}_{4} \mathrm{CO}_{4}$ mainly vibrate along the $a$ direction, and the vibrations along the $b$ and $c$ directions are very slight. This result can be attributed to the fact that we apply the shear strain along the $a$ direction in the ideal strength calculations. Meanwhile, due to the displacement of $\mathrm{B}$ and $\mathrm{O}$ atoms along the $a$ direction at sufficiently large deformation, the $\mathrm{B}-\mathrm{O}$ bonds are hence broken, leading to the collapse of the $t-\mathrm{B}_{4} \mathrm{CO}_{4}$ lattice.

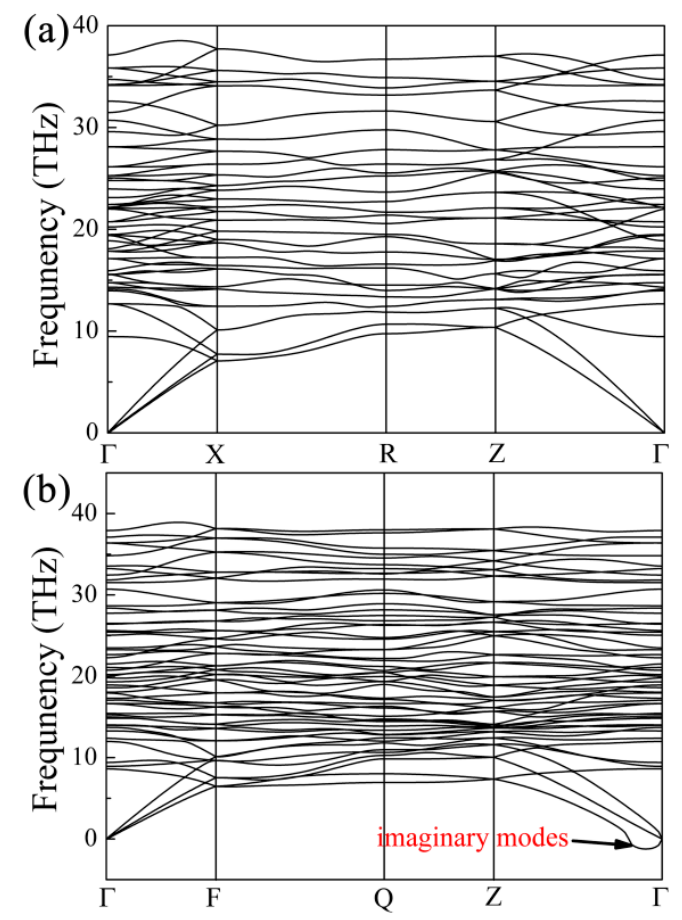

Figure 5. Calculated phonon dispersion curves for $t-\mathrm{B}_{4} \mathrm{CO}_{4}$ before (a) and after (b) shear deformation. 
Note that the minimum ideal shear strength of $t-\mathrm{B}_{4} \mathrm{CO}_{4}$ can only reach $27.5 \mathrm{GPa}$, which is much lower than the superhard criterion of $40 \mathrm{GPa}$, as well as the other ternary B-C-O compounds (See Table 1), suggesting that all these ternary B-C-O compounds currently known may not be intrinsically superhard in spite of their relatively high elastic moduli. The atomic explanation that the ternary B-C-O compounds cannot acquire higher hardness is completely due to the weak B-O bonds being easy to break without exception. Thus, we believe that the possible routes to design the superhard B-C-O compounds can be summarized as follows: (1) one is to reduce the proportion of weak $\mathrm{B}-\mathrm{O}$ ionic bonds and increase the proportion of strong B-C covalent bonds as much as possible in ternary B-C-O compounds; (2) the other is to design special polyhedral crystal structures with less multi-center bridge bonds to resist the shear strain, such as $\mathrm{B}_{12}$ icosahedra in $\mathrm{B}_{6} \mathrm{O}$.

\section{Conclusions}

In summary, we have systematically investigated the mechanical properties of $t-\mathrm{B}_{4} \mathrm{CO}_{4}$, including the elastic constants, bulk modulus, Young's modulus, and shear modulus, in comparison with other ternary B-C-O compounds. The elastic anisotropies of $t-\mathrm{B}_{4} \mathrm{CO}_{4}$ are illustrated according to the analytical formulas of the Young's and shear moduli along different crystal orientations. To examine the superhard feature of $t-\mathrm{B}_{4} \mathrm{CO}_{4}$, the ideal strengths of $t-\mathrm{B}_{4} \mathrm{CO}_{4}$ are estimated based on the strain-stress method. The dominating ideal strength of $27.5 \mathrm{GPa}$ is found along the (001)[100] slip system, indicating that the structural collapse of $t-\mathrm{B}_{4} \mathrm{CO}_{4}$ is far from $40 \mathrm{GPa}$. Thus, we can conclude that $t-\mathrm{B}_{4} \mathrm{CO}_{4}$ is not intrinsically superhard, but is indeed a hard material. We expect that the present work can establish an appropriate approach to distinguish whether a material is superhard, and provide new routes to design the novel superhard B-C-O compounds.

Supplementary Materials: The following are available online at www.mdpi.com/1996-1944/10/2/128/s1. Table S1: The convergence tests of exchange-correlation functional, Table S2: The convergence tests of cutoff energy and $k$-point mesh, Table S3: The convergence tests of the width of the smearing, Table S4: The convergence tests of cutoff energy and $k$-point mesh for ideal strength calculations.

Acknowledgments: The work was financially supported by the Natural Science Foundation of China (Grant Nos.: 11404008 and 11647007), Natural Science Basic Research plan in Shaanxi Province of China (Grant No.: 2016JM106), Education Committee Natural Science Foundation in Shaanxi Province of China (Grant No.: 16JK1049), Scientific Research Program in Baoji City of China (Grant No.: 16RKX1-15), Young Talent fund of University Association for Science and Technology in Shaanxi of China (Grant No.: 20150204), and the Baoji University of Arts and Sciences Key Research (Grant No.: ZK16067).

Author Contributions: Baobing Zheng designed the project and performed the calculations; Baobing Zheng and Meiguang Zhang analyzed the results; Baobing Zheng, Meiguang Zhang, and Canjun Wang wrote the paper.

Conflicts of Interest: The authors declare no conflict of interest.

\section{References}

1. Tian, Y.; Xu, B.; Zhao, Z. Microscopic theory of hardness and design of novel superhard crystals. Int. J. Refract. Met. Hard Mater. 2012, 33, 93-106. [CrossRef]

2. Chung, H.-Y.; Weinberger, M.B.; Levine, J.B.; Kavner, A.; Yang, J.-M.; Tolbert, S.H.; Kaner, R.B. Synthesis of Ultra-Incompressible Superhard Rhenium Diboride at Ambient Pressure. Science 2007, 316, 436-439. [CrossRef] [PubMed]

3. Cumberland, R.W.; Weinberger, M.B.; Gilman, J.J.; Clark, S.M.; Tolbert, S.H.; Kaner, R.B. Osmium Diboride, An Ultra-Incompressible, Hard Material. J. Am. Chem. Soc. 2005, 127, 7264-7265. [CrossRef] [PubMed]

4. Mohammadi, R.; Lech, A.T.; Xie, M.; Weaver, B.E.; Yeung, M.T.; Tolbert, S.H.; Kaner, R.B. Tungsten tetraboride, an inexpensive superhard material. Proc. Natl. Acad. Sci. USA 2011, 108, 10958-10962. [CrossRef] [PubMed]

5. Lech, A.T.; Turner, C.L.; Mohammadi, R.; Tolbert, S.H.; Kaner, R.B. Structure of superhard tungsten tetraboride: $\mathrm{A}$ missing link between $\mathrm{MB}_{2}$ and $\mathrm{MB}_{12}$ higher borides. Proc. Natl. Acad. Sci. USA 2015, 112, 3223-3228. [CrossRef] [PubMed] 
6. Gou, H.; Dubrovinskaia, N.; Bykova, E.; Tsirlin, A.A.; Kasinathan, D.; Schnelle, W.; Richter, A.; Merlini, M.; Hanfland, M.; Abakumov, A.M.; et al. Discovery of a Superhard Iron Tetraboride Superconductor. Phys. Rev. Lett. 2013, 111, 157002. [CrossRef] [PubMed]

7. Kumar, N.R.S.; Sharat, C.; Amirthapandian, S.; Shekar, N.V.C.; Sahu, P.C. Investigations of the high pressure synthesized osmium carbide by experimental and computational techniques. Mater. Res. Express 2015, 2, 016503. [CrossRef]

8. Crowhurst, J.C.; Goncharov, A.F.; Sadigh, B.; Evans, C.L.; Morrall, P.G.; Ferreira, J.L.; Nelson, A.J. Synthesis and Characterization of the Nitrides of Platinum and Iridium. Science 2006, 311, 1275-1278. [CrossRef] [PubMed]

9. Young, A.F.; Sanloup, C.; Gregoryanz, E.; Scandolo, S.; Hemley, R.J.; Mao, H.-K. Synthesis of Novel Transition Metal Nitrides $\mathrm{IrN}_{2}$ and $\mathrm{OsN}_{2}$. Phys. Rev. Lett. 2006, 96, 155501. [CrossRef] [PubMed]

10. Qin, J.; He, D.; Wang, J.; Fang, L.; Lei, L.; Li, Y.; Hu, J.; Kou, Z.; Bi, Y. Is Rhenium Diboride a Superhard Material? Adv. Mater. 2008, 20, 4780-4783. [CrossRef]

11. Yang, J.; Sun, H.; Chen, C. Is Osmium Diboride An Ultra-Hard Material? J. Am. Chem. Soc. 2008, 130, 7200-7201. [CrossRef] [PubMed]

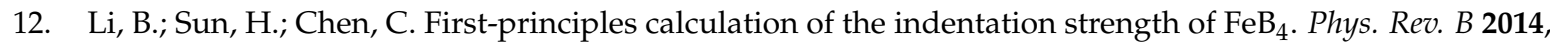
90, 014106. [CrossRef]

13. Zhang, M.; Lu, M.; Du, Y.; Gao, L.; Lu, C.; Liu, H. Hardness of FeB 4 : Density functional theory investigation. J. Chem. Phys. 2014, 140, 174505. [CrossRef] [PubMed]

14. Zinin, P.V.; Ming, L.C.; Ishii, H.A.; Jia, R.; Acosta, T.; Hellebrand, E. Phase transition in BC $_{x}$ system under high-pressure and high-temperature: Synthesis of cubic dense $\mathrm{BC}_{3}$ nanostructured phase. J. Appl. Phys. 2012, 111, 114905. [CrossRef]

15. Solozhenko, V.L.; Kurakevych, O.O.; Andrault, D.; Le Godec, Y.; Mezouar, M. Ultimate Metastable Solubility

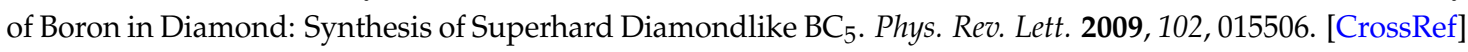
[PubMed]

16. Solozhenko, V.L.; Andrault, D.; Fiquet, G.; Mezouar, M.; Rubie, D.C. Synthesis of superhard cubic BC ${ }_{2}$ N. Appl. Phys. Lett. 2001, 78, 1385-1387. [CrossRef]

17. Badzian, A.R. Superhard material comparable in hardness to diamond. Appl. Phys. Lett. 1988, 53, $2495-2497$. [CrossRef]

18. Garvie, L.A.J.; Hubert, H.; Petuskey, W.T.; McMillan, P.F.; Buseck, P.R. High-Pressure, High-Temperature syntheses in the B-C-N-O system. J. Solid State Chem. 1997, 133, 365-371. [CrossRef]

19. Bolotina, N.B.; Dyuzheva, T.I.; Bendeliani, N.A. Atomic structure of boron suboxycarbide $\mathrm{B}(\mathrm{C}, \mathrm{O})_{0.155}$. Crystallogr. Rep. 2001, 46, 734-740. [CrossRef]

20. Gladkaya, I.S.; Dyuzheva, T.I.; Ekimov, E.A.; Nikolaev, N.A.; Bendeliani, N.A. Crystal growth at high pressure and the problem of characterization of the interstitial phases in the B-C-O system. J. Alloys Compd. 2001, 329, 153-156. [CrossRef]

21. Broitman, E.; Gueorguiev, G.K.; Furlan, A.; Son, N.T.; Gellman, A.J.; Stafström, S.; Hultman, L. Water adsorption on fullerene-like carbon nitride overcoats. Thin Solid Film. 2008, 517, 1106-1110. [CrossRef]

22. Gueorguiev, G.K.; Czigány, Z.; Furlan, A.; Stafström, S.; Hultman, L. Intercalation of P atoms in Fullerene-like $\mathrm{CP}_{\mathrm{x}}$. Chem. Phys. Lett. 2011, 501, 400-403. [CrossRef]

23. Hellgren, N.; Berlind, T.; Gueorguiev, G.K.; Johansson, M.P.; Stafström, S.; Hultman, L. Fullerene-like BCN thin films: A computational and experimental study. Mater. Sci. Eng. B 2004, 113, 242-247. [CrossRef]

24. Yinwei, L.; Quan, L.; Yanming, M. B 2 CO: A potential superhard material in the B-C-O system. Europhys. Lett. 2011, 95, 66006.

25. Liu, C.; Zhao, Z.; Luo, K.; Hu, M.; Ma, M.; He, J. Superhard orthorhombic phase of $\mathrm{B}_{2} \mathrm{CO}$ compound. Diam. Relat. Mater. 2016. [CrossRef]

26. Zhang, M.; Yan, H.; Zheng, B.; Wei, Q. Influences of carbon concentration on crystal structures and ideal strengths of $\mathrm{B}_{2} \mathrm{C}_{\mathrm{x}} \mathrm{O}$ compounds in the B-C-O system. Sci. Rep. 2015, 5, 15481. [CrossRef] [PubMed]

27. Wang, S.; Oganov, A.R.; Qian, G.; Zhu, Q.; Dong, H.; Dong, X.; Davari Esfahani, M.M. Novel superhard B-C-O phases predicted from first principles. Phys. Chem. Chem. Phys. 2016, 18, 1859-1863. [CrossRef] [PubMed]

28. Perdew, J.P.; Burke, K.; Ernzerhof, M. Generalized Gradient Approximation Made Simple. Phys. Rev. Lett. 1996, 77, 3865-3868. [CrossRef] [PubMed] 
29. Kresse, G.; Furthmüller, J. Efficient iterative schemes for ab initio total-energy calculations using a plane-wave basis set. Phys. Rev. B 1996, 54, 11169-11186. [CrossRef]

30. Kresse, G.; Joubert, D. From ultrasoft pseudopotentials to the projector augmented-wave method. Phys. Rev. B 1999, 59, 1758-1775. [CrossRef]

31. Monkhorst, H.J.; Pack, J.D. Special points for Brillouin-zone integrations. Phys. Rev. B 1976, 13, 5188-5192. [CrossRef]

32. Hill, R. The Elastic Behaviour of a Crystalline Aggregate. Proc. Phys. Soc. Lond. Sect. A 1952, 65, 349. [CrossRef]

33. Roundy, D.; Krenn, C.R.; Cohen, M.L.; Morris, J.W. Ideal Shear Strengths of fcc Aluminum and Copper. Phys. Rev. Lett. 1999, 82, 2713-2716. [CrossRef]

34. Zhang, R.F.; Sheng, S.H.; Veprek, S. First principles studies of ideal strength and bonding nature of AlN polymorphs in comparison to TiN. Appl. Phys. Lett. 2007, 91, 031906. [CrossRef]

35. Zhang, R.F.; Veprek, S.; Argon, A.S. Anisotropic ideal strengths and chemical bonding of wurtzite BN in comparison to zincblende BN. Phys. Rev. B 2008, 77, 172103. [CrossRef]

36. Wang, Y.-J.; Wang, C.-Y. Mechanical properties and electronic structure of superhard diamondlike $\mathrm{BC}_{5}$ : A first-principles study. J. Appl. Phys. 2009, 106, 043513. [CrossRef]

37. Zhang, R.F.; Lin, Z.J.; Veprek, S. Anisotropic ideal strengths of superhard monoclinic and tetragonal carbon and their electronic origin. Phys. Rev. B 2011, 83, 155452. [CrossRef]

38. Mouhat, F.; Coudert, F.-X. Necessary and sufficient elastic stability conditions in various crystal systems. Phys. Rev. B 2014, 90, 224104. [CrossRef]

39. Kelly, A.; MacMillan, N.H. Strong Solids, 3rd ed.; Oxford University Press: Oxford, UK, 1986.

40. Lyakhov, A.O.; Oganov, A.R. Evolutionary search for superhard materials: Methodology and applications to forms of carbon and $\mathrm{TiO}_{2}$. Phys. Rev. B 2011, 84, 092103. [CrossRef]

41. Chen, X.-Q.; Niu, H.; Li, D.; Li, Y. Modeling hardness of polycrystalline materials and bulk metallic glasses. Intermetallics 2011, 19, 1275-1281. [CrossRef]

42. Veprek, S.; Zhang, R.F.; Argon, A.S. Mechanical properties and hardness of boron and boron-rich solids. J. Superhard Mater. 2011, 33, 409-420. [CrossRef]

(C) 2017 by the authors; licensee MDPI, Basel, Switzerland. This article is an open access article distributed under the terms and conditions of the Creative Commons Attribution (CC BY) license (http:/ / creativecommons.org/licenses/by/4.0/). 